\section{Very rapid forgetting: Reply to Muter}

\author{
ALICE F. HEALY \\ University of Colorado, Boulder, Colorado \\ and \\ THOMAS F. CUNNINGHAM \\ St. Lawrence University, Canton, New York
}

We reply to the commentary in which Muter (1995) disputes the findings and arguments of Cunningham, Healy, Till, Fendrich, and Dimitry (1993) concerning the rate of forgetting from primary memory. Although Muter (1980) had reported very rapid forgetting when secondary memory processes were minimized, Cunningham et al. found that the rate of forgetting from primary memory was no more rapid when secondary memory processes were minimal than it was when secondary memory processes were substantial. We locate the discrepancy between the two studies specifically at recall performance on the 0-sec retention interval, and we argue that the very rapid forgetting found by Muter (1980) can be attributed in large part to the fact that although subjects in his experiments did not expect to recall the letters after a filled retention interval, at the time of encoding and after an unfilled delay they did have a high overall expectancy to recall the letters.

We found Muter's (1995) commentary to be stimulating and challenging. Although we disagree with much of what Muter says, the issues and criticisms that he raises with regard to our (Cunningham, Healy, Till, Fendrich, \& Dimitry, 1993) goals, procedures, findings, and conclusions illustrate well the complexities involved in this area of research. We believe that the concept of very rapid forgetting in short-term memory has been oversimplified in the past and that a close examination of our study visà-vis Muter's commentary will help clarify the conceptual and procedural complexities and confusions associated with the phenomenon.

In his commentary, Muter (1995) argues that the experiments reported by Cunningham et al. (1993) do not constitute failures to replicate Muter's (1980) experiments demonstrating very rapid forgetting. Muter (1995) posits that Cunningham et al.'s procedures lack some of the necessary conditions for studying forgetting from primary memory and that their data do not cast doubt on the finding of very rapid forgetting. More specifically, Muter (1995) cites as two major problems the expectations of the subjects with regard to recall and the diffi-

Preparation of this article was supported in part by Army Research Institute Contract MDA903-93-K-0010 to the University of Colorado. Correspondence concerning this article should be addressed either to A. F. Healy, Department of Psychology, University of Colorado, Campus Box 345, Muenzinger Building, Boulder, CO 80309-0345 or to T. F. Cunningham, Department of Psychology, St. Lawrence University, Flint Hall, Canton, NY 13617 (e-mail: ahealy@clipr.colorado.edu and tcun@music.stlawu.edu, respectively). culty level of the distractor task. According to Muter (1995), when Cunningham et al.'s experiments are compared with those of Muter (1980), the recall expectancy of the subjects is higher and the distractor task is easier in the experiments of Cunningham et al. Muter (1995) also throws doubt on the findings of Cunningham et al. by posing the question of whether the to-be-remembered items in those experiments were ever registered in the subjects' memory and by pointing out that Cunningham et al.'s experiments tap only memory for order information, not both item and order information as in the earlier study by Muter (1980). Finally, Muter (1995) presents a new figure with data from Cunningham et al.'s study suggesting that despite these differences forgetting was more rapid when importance was low, contrary to the claims of Cunningham et al. In the present reply, we respond to each of these arguments in turn.

\section{Goals of the Study by Cunningham et al. (1993)}

The phenomenon of very rapid forgetting discovered by Muter (1980) is of crucial importance to our understanding of short-term memory processes and has had a substantial impact on the field. For example, this work is cited prominently in an encyclopedia article by Logan (1992) on attention and memory. This phenomenon was successfully replicated by Sebrechts, Marsh, and Seamon (1989). It was not Cunningham et al.'s (1993) intention to attempt to provide another replication of this phenomenon. In fact, there were reasons of major importance why a different experimental design was chosen for the Cunningham et al. experiments. Hence, we find inappropriate the major premise of the commentary by Muter (1995) - that is, the assumption that the experiments of Cunningham et al. represented an attempt to replicate Muter's (1980) findings (see Muter, 1995, p. 383).

In the introduction to our earlier article, we (Cunningham et al., 1993) reviewed two lines of investigation. The first was the one initiated by Muter (1980) and the second was introduced by Healy, Fendrich, Cunningham, and Till (1987). Both lines of investigation use variants of the distractor paradigm and both

indicate that the subjects' expectancy concerning test procedure plays an important role in the retention functions obtained in the distractor paradigm, with recall depressed when expectancy is reduced in both cases. The overall rapid rate of forgetting found in the first line of investigation raises the interesting question of whether the rate of forgetting from primary memory is affected by expectancy. The results of the second line of investigation suggest that there is no change in the rate of forgetting from primary memory when there is a reduction in the contribution of secondary memory processes. However, the reduction of secondary memory processes was modest in the second line of investigation relative to that attained in the first line of investigation. (Cunningham et al., 1993, p. 672) 
Hence, the major goal of the study by Cunningham et al. (1993) was to

continue the second line of investigation, in an attempt to achieve a more powerful reduction of secondary memory processes and to examine the rate of forgetting from primary memory when primary memory processes are isolated as much as possible. (p. 672)

In his commentary, Muter (1995) quotes this goal (see p. 385) but cites only part of it out of context, so that he does not make it clear that it was of crucial concern in the investigation of Cunningham et al. (1993) to build on the experimental and theoretical methods developed by Healy et al. (1987) and to see whether very rapid forgetting would be obtained with those methods, rather than only with the ones developed by Muter (1980).

\section{Recall Expectancy}

Establishing the rate of forgetting in short-term memory when secondary memory processes are minimized or absent is important and was in fact a goal of both the study by Muter (1980) and the subsequent study by Cunningham et al. (1993). Unfortunately, Muter (1980) did not obtain a satisfactory answer to this problem, because in his paradigm the recall expectancy of his subjects at time of encoding (and 0 -sec retention interval) was very high, certainly much higher than that present in the Cunningham et al. (1993) experiments wherein subjects were misled prior to the time of encoding regarding which of two four-letter segments was important for recall.

It is critical to discriminate between Muter's (1980) experiments and our experiments in terms of when recall expectancy should be low and, hence, secondary memory processing minimized. In Cunningham et al.'s (1993) experiments, recall expectancy is low both at the time of initial encoding and throughout the retention intervals associated with subsequent tests of recall. Such is not the case in Muter's (1980) paradigm. There is, indeed, every reason to believe that recall expectancy and secondary memory processing are present at different levels at the time of encoding and at the time of subsequent testing following distractor-filled retention intervals.

Recall expectancy in the study by Muter (1980). Muter (1995) claims that "expectancy of recall with a 0 -sec interval was low" (p. 385) in the study by Muter (1980). Strictly speaking, this claim has some validity, because subjects were rarely tested at a 0 -sec interval. However, the majority of their trials were "maintenance" trials, which were essentially equivalent to critical test trials at a 0 -sec interval. Hence, here lies a major confusion on Muter's part as well as on the part of other researchers in the field. For example, in the encyclopedia article already mentioned, Logan (1992) states, "Muter (1980) found complete decay within 3 seconds if people did not expect to have to recall the memory list" (pp. 64 65). Likewise, Sebrechts et al. state, "in the Muter paradigm, subjects are not expecting a recall task" (p. 695). However, subjects in Muter's (1980) experiments had in fact a very high expectancy to recall the memory list of letters. Muter (1995) himself admits that "expectancy of recall with an unfilled retention interval greater than zero was high" (p. 385) in the Muter (1980) experiments. If subjects expect to recall the to-be-remembered items after a brief ( $2 \mathrm{sec}$ or less) unfilled retention interval, they are certainly prepared to recall the list immediately (i.e., with a 0 -sec interval) even if they have not previously been asked to do so.

Muter (1980) attempted to reduce the effect of secondary memory processing on to-be-tested material by leading subjects to expect that they would never, or rarely, be tested for recall of target material (trigrams) following activity on a distractor task. He led subjects to this expectation in two ways. First, he introduced trials in which subjects received practice on a distractor task without a subsequent test of the target material. These trials, which were called "counting" or "reading" trials, accounted for $17 \%$ of the experimental trials in Experiment 1 and $9 \%$ of the experimental trials in Experiment 2 of Muter (1980). The basic idea was that practice on these trials - that is, trials with no recall test after distractor activity - would serve to lower recall expectancy on the critical test trials and thus lead the subject to engage in nonelaborative rehearsal of target material. There is no argument with Muter (1980) in that the presence of a distractor-filled retention interval should serve as an effective cue for reducing recall expectancy (and secondary memory rehearsal processes) and produce low recall on critical test trials of 2,4 , or $8 \mathrm{sec}$.

A second way that Muter (1980) attempted to reduce secondary memory processing of to-be-tested material on critical test trials was to use "maintenance" trialsthat is, trials in which subjects received a trigram for $1 \mathrm{sec}$, a blank screen for $2 \mathrm{sec}$ (or $1.5 \mathrm{sec}$ in Experiment 2), and a word prompt, LETTERS, that acted as a cue for recall of the trigram presented at the start of the trial. The percentage of trials in which trigrams were tested following a 2-or a 1.5-sec interval of no distractor activity was $78 \%$ in Muter's Experiment 1 and $84 \%$ in his Experiment 2. Presumably, Muter would argue that because there was no distractor activity the memory task was a relatively effortless one that minimized the need to use secondary or elaborative processing.

We take issue with the assumption that the "maintenance" trials are effective in producing nonelaborative processing at the time of encoding and immediate testing (i.e., the 0 -sec retention interval) on Muter's critical trials. Muter (1980) offers no empirical evidence to indicate that only nonelaborative processing is taking place. In fact, the proportion of trigrams recalled correctly on the maintenance trials and the critical trials at the 0 -sec retention interval was $90 \%$ or greater for Muter (1980), which is comparable to the percentage reported in the traditional work of Peterson and Peterson (1959) and Murdock (1961), in which recall expectancy was undoubtedly high and, hence, secondary or elaborative processing was likely. Muter (1995) himself points out, "performance at the 0-sec interval in Muter was similar to that in Murdock (1961)" (p. 385), in which recall was 
expected by subjects on every trial. Furthermore, Sebrechts et al. (1989) have demonstrated that orienting tasks can influence recall in both the traditional distractor paradigm and the paradigm introduced by Muter (1980); they found "the presence of secondary memory influences at even the shortest retention interval" (Sebrechts et al., 1989, p. 693), which is why they speculated that "forgetting from primary memory may be even more rapid than has been proposed" (p. 693).

Thus, the high overall recall expectancy by the subjects can explain why Muter (1980) found very high performance both on his maintenance trials and on his critical trials occurring after a 0 -sec retention interval (which were essentially equivalent to the maintenance trials).

One thing that was clearly unexpected by Muter's (1980) subjects was that they would recall the letters after an interval filled with the distractor task (the counting backwards task in Experiment 1 or the word reading distractor in Experiment 2), because in Muter's (1980) paradigm the distractor task was typically not followed by recall of the list of letters. For that reason Muter (1980) found very low performance on his critical trials occurring after $4 \mathrm{sec}$ of an interval filled with the counting task in Experiment 1 or after 2,4 , and $8 \mathrm{sec}$ of an interval filled with the word reading task in Experiment 2. Thus, the distractor tasks in Muter's (1980) experiments served a function not unlike that of the forget cues in the studies of directed forgetting (see, e.g., Bjork, 1972, and Muter, 1980 , p. 178), but they did nothing to change the subjects' initial encoding of the to-be-remembered letters.

We would thus argue that the very rapid rate of forgetting reported by Muter (1980) is an artifact produced by differences in recall expectancy between the 0 -sec retention test and later tests. The paradigm used by Muter (1980) encourages subjects to encode strongly the trigram material on every experimental trial in which such material is presented. This encouragement occurs because the number of trials in which trigrams are subsequently tested for recall is so great. It is extremely likely that any subject with a history involving a request for recall of trigrams on approximately $80 \%$ or more of the experimental trials would have a high expectancy at the time of initial encoding that the to-be-presented items would have to be recalled. This high level of recall expectancy is not altered prior to or during a 0 -sec recall test, but it is markedly reduced by the onset of distractor activity that occurs in later recall tests. It is only after the presentation of the trigram and the immediate presentation of distractor activity that a subject's recall expectancy is reduced. Cunningham et al.'s (1993) point was that subjects in Muter's (1980) experiments expected to recall the letters at the 0 -sec interval but not after the 2 -sec or longer intervals of distracting activity so that "delay interval was confounded with expectancy to recall" (Cunningham et al., 1993, p. 683). In his commentary, Muter (1995) notes that "even in Muter's experiments, there was no evidence of very rapid forgetting beyond a $2-\mathrm{sec}$ retention interval" (p. 384). Note that $2 \mathrm{sec}$ is in fact the shortest filled retention interval used by Muter on the critical trials. Thus, the evidence of very rapid forgetting found by Muter comes entirely from the comparison of the 0 -sec retention interval critical trials (which are consistent with the subjects' overall expectations that they are to recall the to-be-remembered letters) with the 2-sec or longer retention interval critical trials (in which subjects have a distractor task which is normally not followed by recall of the to-be-remembered letters so those trials are inconsistent with the subjects' specific expectations that they would not have to recall the letters after an interval of distracting activity).

Recall expectancy in the study by Cunningham et al. (1993). On the other hand, in the experiments by Cunningham et al. (1993), encoding processes were specifically manipulated so as "to reduce the operation of secondary-memory encoding processes" (Cunningham et al., 1993, p. 673). This manipulation was achieved in Experiment 1 by varying the subjects' expectancy for a particular recall test (subjects in the crucial 75\% precue condition were led by a precue to expect to recall one of the to-be-remembered four-letter segments but then on $25 \%$ of the trials were forced by a postcue to switch recall to the other segment instead). This manipulation was achieved in Experiment 2 by varying the importance of the material to be recalled (subjects were led to believe that the crucial noncued "distractor" segment was less important to recall than the cued "target" segment even though the distractor segment was to be recalled first). The fact that both of these manipulations of encoding processes were successful in the experiments of Cunningham et al. (1993) is underlined by the large effect of the switch manipulation in Experiment 1 (see Cunningham et al., 1993, pp. 676-677) and by the sizable interaction of cue condition and the distractor/target distinction in Experiment 2 (see p. 680, and note that performance was superior on the target segment than on the distractor segment in both cuing conditions, even though the distractor segment was recalled first).

Summary. We believe that it is reasonable to assume that the 0 -sec critical trial in the Muter (1980) paradigm has the same recall expectancy associated with it as would be found in using the procedure of Peterson and Peterson (1959), in which subjects expect to be tested for recall on every trial and, hence, secondary or elaborative processing is likely. Recall should have been high for Muter (1980) on the 0-sec critical trial. And it was. What created the precipitous drop in performance in the Muter (1980) paradigm was the differential recall expectancy at the 0 -sec critical trial and the other critical trials which occur at 2,4 , or $8 \mathrm{sec}$ following a distractor-filled retention interval.

The procedures used by Cunningham et al. (1993) were designed to affect the type of processing engaged in at the time of initial encoding as well as at later test periods ranging from immediate recall to delay periods of up to $12 \mathrm{sec}$. We achieved this effect either by inducing a low recall expectancy prior to stimulus presentation or by emphasizing the unimportant nature of the material prior to stimulus presentation. The fact that Cunningham et al. 
did succeed in this endeavor is evident in the low level of recall obtained at the 0 -sec retention test as well as at the other retention test intervals in Experiment 2 of that study. This observation accounts for why Cunningham et al. (1993) did not obtain a precipitous drop, as Muter (1980) did, from the 0-sec test to later tests and why Cunningham et al., therefore, did find a forgetting rate comparable to that found in traditional studies using the Peterson and Peterson (1959) paradigm.

\section{Distractor Tasks}

Muter (1995) is concerned that the distractor task used by Cunningham et al. (1993) was too easy. We do not appreciate the significance of this concern, because the rate of forgetting curves reported by Muter (1980) and Cunningham et al. differ most markedly in performance at the 0 -sec retention test when no distractor activity occurs. In both studies, recall performance was relatively low at subsequent retention tests following distractor activity. Nevertheless, Muter (1995) is correct that the digit shadowing distractor task used by Cunningham et al. (1993) was different from, and perhaps easier than, the counting backward and word reading tasks used by Muter (1980), but the digit shadowing task was used for at least two good reasons. First, the digit shadowing distractor task was the one used in the earlier study by Healy et al. (1987) as well as in the previous work on which that study was based (see Lee \& Estes, 1981, and Cunningham, Healy, \& Williams, 1984). Second, that task provided control by the experimenter over the rate of the subject's responses (each digit is presented at the rate of one per $1 / 2 \mathrm{sec}$ in the fast presentation rate or one per sec in the slow presentation rate, so that the subject must respond at that rate), whereas no such control is provided over the rate of the counting backward and word reading tasks used by Muter (1980). Such control over the rate of the distractor task was necessary, because mathematical modeling using the Estes (1972; Lee \& Estes, 1981) perturbation model was the primary theoretical tool employed by Cunningham et al. (1993) and because that modeling requires an assumption about the number of reactivations (or chances for perturbations) that occur during the distractor task (the specific assumption is made that there are two reactivations for each digit presented during the digit shadowing distractor task; see Cunningham et al., 1993, p. 685).

Although the distractor task used by Cunningham et al. (1993) may be easier than the counting backward or word reading distractor tasks used by Muter (1980), the memory task used by Cunningham et al. (1993) was undoubtedly more difficult than the one used by Muter (1980) if memory load is considered in assessing the difficulty of the memory task. The memory load imposed on subjects in Cunningham et al.'s (1993) study is considerably greater than that imposed on subjects in Muter's (1980) study. For example, in Muter's (1980) Experiment 1 , a single trigram (three consonants) was read silently and was recalled following a distractor task of counting backward by threes. In Cunningham et al.'s (1993) Experiment 2, subjects were required to recall two four-letter segments with the letters within each segment to be recalled in their correct order. In any event, whether or not the distractor task used by Cunningham et al. (1993) is easier than Muter's (1980), there is no question that it was successful, as is evident from the extremely large effects of retention interval in both Experiment 1 (see Cunningham et al., 1993, p. 676) and Experiment 2 (see Cunningham et al., 1993, p. 680).

\section{Registering Items in Primary Memory}

Because initial performance (i.e., at the 0 -sec retention interval) was so poor in the study by Cunningham et al. (1993), in his commentary Muter (1995) questions "whether the four to-be-remembered items were ever solidly in primary memory together at any one time" (p. 385). In Muter's (1980) experiments, subjects silently read the to-be-remembered letters which were presented together at the start of a trial. However, in the experiments by Cunningham et al. (1993), subjects were required to read aloud each to-be-remembered letter as it was individually presented on the display screen. An "experimenter remained by the subject's side to ensure that the subjects read aloud all characters as they appeared on the display monitor" (Cunningham et al., 1993, p. 674). Hence, there was no question that the to-beremembered letters were solidly registered in primary memory in the experiments by Cunningham et al. Furthermore, the initial performance in the study by Cunningham et al. was no lower than that in the study by Sebrechts et al. (1989) with the acoustic orienting task (see p. 698). A crucial finding in Cunningham et al.'s study is that when secondary memory encoding processes are reduced, memory performance is low even at the initial $(0-\mathrm{sec})$ retention interval. Cunningham et al. (1993) also show (see p. 687) that for their conditions in which secondary memory encoding processes are not reduced, their results are consistent with those from the comparable conditions of their earlier study (Healy et al., 1987). In that earlier study, performance at the 0 -sec retention interval was considerably higher (e.g., recall of the second segment was approximately $90 \%$ correct at the 0 -sec retention interval in the precue condition of Experiment 1; see Healy et al., 1987, p. 415).

\section{Order and Item Information}

In his commentary, Muter (1995) also complains that Cunningham et al.'s (1993) experiments "tap only memory for order information" and speculates that "perhaps forgetting rates are also different for order and item information" (p. 385). That point seems to be well addressed by Cunningham et al. (1993). For example, they stated,

It is possible that the rate of forgetting from primary memory is more rapid for item information than for order information. However, the rapid forgetting found by Muter and Sebrechts et al. was most evident with their strict scoring method, which scored for order as well as item in- 
formation, rather than with their lenient scoring method, which scored only for item information. (p. 687)

See also note 2 in the commentary by Muter (1995).

\section{New Figure}

Finally, Muter (1995) in his commentary presents a new figure (Figure 1) based on data from Experiment 2 of the study by Cunningham et al. (1993). Specifically, Figure 1 displays proportion correct as a function of retention interval for the target and distractor conditions in the precue condition only, for the second segment only, collapsed across exposure rate and serial position. This figure, which is based on means provided in Table 2 of the report by Cunningham et al. (1993), suggests a faster forgetting rate in the distractor condition than in the target condition. However, if one returns to Table 2 of the report by Cunningham et al. (1993), it is evident that the difference in forgetting rate is most pronounced for the slow exposure rate (which allows secondary memory encoding processes, distractor: 0-digit, .771; 4-digit, .552; 12-digit, .469; target: 0-digit, .781; 4-digit, .677; 12-digit, .651) and is considerably reduced for the more crucial fast exposure rate (which minimizes secondary memory encoding processes, distractor: 0 -digit, .630; 4digit, .406; 12-digit, .339; target: 0 -digit, .635; 4-digit, $.495 ; 12$-digit, .430 ). Therefore, the data underlying Figure 1 do not substantiate Muter's (1995) conjecture that very rapid forgetting may have taken place in our Experiment 2 when secondary memory was minimized.

In any event, there does not seem to be sufficient justification for examining only the subset of data included in Figure 1 (i.e., the second segment collapsed over exposure rate). The figure included by Cunningham et al. (1993) (Figure 4, p. 681) reflected a small but significant three-way interaction of segment status, segment position, and retention interval, and Cunningham et al. (1993) point out that the crucial three-way interaction of retention interval, segment status, and cue condition was not significant (see p. 681). (It may also be interesting to note that neither the four-way interaction of retention interval, segment status, cue condition, and segment position nor the five-way interaction of exposure rate, retention interval, segment status, cue condition, and segment position approached significance.) Note that despite the trends evident in Muter's (1995) Figure 1, Cunningham et al. (1993) were able to fit their data with the Estes (1972; Lee \& Estes, 1981) perturbation model by using a single constant value of theta (the parameter reflecting the rate of forgetting from primary memory) for both the distractor and the target segments at both the fast and the slow presentation rates, varying only alpha (the secondary memory encoding parameter; see pp. 686-687).

\section{Conclusions}

It is important to distinguish between an overall expectancy to recall and a specific expectancy to recall after a period of distracting activity. Muter's (1980) earlier experiments induced a high overall expectancy to recall but a low specific expectancy to recall after a filled retention interval. As a result, Muter (1980) found extremely high recall performance after an unfilled or 0 sec retention interval but low recall performance after a filled retention interval. In contrast, in the experiments by Cunningham et al. (1993), we induced a low overall expectancy to recall along with a low specific expectancy to recail after a filled retention interval. Consequently, we found low recall performance both after a 0 -sec retention interval and after a filled retention interval. These different findings led to very different conclusions about the rate of forgetting from primary memory when secondary memory processes are minimized. Although Muter (1980) concluded that the rate of forgetting was very rapid, we concluded that the rate of forgetting was no more rapid than in previous studies in which secondary memory processes were evident (e.g., Cunningham et al., 1984; Healy et al., 1987; Lee \& Estes, 1981). Muter (1995) expresses surprise (see p. 385) that subjects would ever exhibit difficulty in recalling a small segment of letters immediately after their presentation (i.e., at a 0 -sec retention interval). However, in the experiments by Cunningham et al. (1993), we found that recall performance was in fact depressed under those conditions provided that subjects were induced to give greater importance to their subsequent recall of another segment of letters shown on the same trial. The Estes (1972; Lee \& Estes, 1981) perturbation model provided a theoretical framework in which Cunningham et al. (1993) could make sense of this surprising finding. In particular, our fits to the perturbation model suggested that a substantial amount of forgetting occurred while the subjects moved their attention from the important segment to the less important segment which they were to recall first on a given trial.

\section{REFERENCES}

Bjork, R. A. (1972). Theoretical implications of directed forgetting. In A. W. Melton \& E. Martin (Eds.), Coding processes in human memory (pp. 217-235). New York: Halstead.

Cunningham, T. F., Healy, A. F., Till, R. E., Fendrich, D. W., \& Dimitry, C. Z. (1993). Is there really very rapid forgetting from primary memory? The role of expectancy and item importance in short-term recal]. Memory \& Cognition, 21, 671-688

Cunningham, T. F. Healy, A. F., \& Williams, D. M. (1984). Effects of repetition on short-term retention of order information. Journal of Experimental Psychology: Learning, Memory. \& Cognition, 10, 575-597.

Estes, W. K. (1972), An associative basis for coding and organization in memory. In A. W. Melton \& E. Martin (Eds.), Coding processes in human memory (pp. 161-190). New York: Halstead.

Healy, A. F, Fendrich, D. W., Cunningham, T. F., \& Till, R. E. (1987). Effects of cuing on short-term retention of order information. Journal of Experimental Psychology: Learning, Memory, \& Cognition, 13, 413-425

LEE, C. L., \& ESTES, W. K. (1981). Item and order information in shortterm memory: Evidence for multilevel perturbation processes. Journal of Experimental Psychology: Human Learning \& Memory, 7, $149-169$

Logan, G. D. (1992). Attention and memory. In L. R. Squire et al. (Eds.), Encyclopedia of learning and memory (pp. 63-67). New York: Macmillan 
Murdock, B. B., JR. (1961). The retention of individual items. Journal of Experimental Psychology, 62, 61 8-625.

MUTER, P. (1980). Very rapid forgetting. Memory \& Cognition, 8, 174 179.

MUTER, P. (1995). Very rapid forgetting: Reply to Cunningham, Healy, Till, Fendrich, and Dimitry. Memory \& Cognition, 23, 383-386. Peterson, L. R., \& Peterson, M. J. (1959). Short-term retention of individual verbal items. Journal of Experimental Psychology, 58, 193-198.
Sebrechts, M. M., Marsh, R. L., \& Seamon, J. G. (1989). Secondary memory and very rapid forgetting. Memory \& Cognition, 17, 693700 .

(Manuscript received June 4, 1994; revision accepted for publication August 4, 1994.) 